\title{
Alternative Paradigm for the Cosmic Objects Formation: New Prospects
}

\section{H. A. Harutyunian}

NAS RA V. Ambartsumian Byurakan Astrophysical Observatory (BAO), Armenia

E-mail: hhayk@bao.sci.am

\begin{abstract}
We consider here briefly the cosmogonic concept suggested by Viktor Ambartsumian in the last century for explaining the formation of cosmic objects. He grounded his concept using the observational facts available in 40s-60s of the last century. The analysis of observational data allowed him to conclude that cosmic objects formation takes place up to nowadays. The second and more "heretical" conclusion he arrived at persuades that the origination and further evolution of cosmic objects goes on in course of gradual decay of proto-stellar matter at all hierarchical levels. We argue that this approach appeared first time in Ambartsumian's papers devoted to the problems of quantum electrodynamics. Later on his concept on objects formation due to decay of protostellar dense matter was rejected because the known laws of physics do not allow existence of huge masses consisted of superdense matter. We bring to the readers' attention, that the discovery of dark energy changes the situation drastically and opens new rooms for the ideas forgotten by the scientific community.
\end{abstract}

Keywords: Universe expansion - dark energy - stellar associations - active galactic nuclei - atomic nuclei - mass-energy transformation

\section{Introduction}

The scientific community at any epoch disposes of certain storage of knowledge and tools to use the knowledge available. Consequently, every epoch brings into use some amount of the new knowledge. Appearing on the basis of the available for the given epoch knowledge is not surely correct in a broad sense. It only should satisfy the requirement of the self-consistency and credibility within the frame of existing knowledge.

The process of cosmic objects formation was always a subject of debates since the time of ancient philosophers. Newton's discovery of the gravitation low created for the first time for construction of scientifically based hypothesis on the stellar and planetary formation. The first one to suggest a hypothesis on the star formation was Immanuel Kant who put forward an idea that the stars and planets formed due to a 
process of collapsing in of thin and dim clouds of gas and dust on themselves as a result of gravitational interaction between the countless faint particles. According to nowadays cosmogony, stars form in giant molecular clouds, which are gravitationally unstable. Owing to instability, the matter within clouds coalesces into many separate and denser clumps forming stars later on.

Although that was a scientifically based hypothesis for the observational data available for that time, which initiated many new studies, subsequently it became inhibitory for the further progress in this field. Every step of scientific progress in this and related areas inevitably should fit the basic cosmogonic idea. Due to the above dictate, astronomical community believes that almost all the cosmic objects and their systems are gravitationally bound. Moreover, this issue is not disputable, though never proved observationally or theoretically.

Greatest cosmological discoveries of the twentieth century - the Universe expansion and, later on, its accelerated behavior - made the physical picture of the Universe very odd. Following to the adopted ideas on the Universe structure, one arrives at a conclusion that the expanding as a whole, the unique entity of matter, energy and space is composed of embedded cells of various scales collapsing independently. Where is the discontinuity boundary and how it evolves, remains obscure.

One of the oddest creations resulted by Kant's hypothesis and its theoretical modifications is the "dark matter" which was initially invented by Zwicky (1933) for vouching the stability of clusters of galaxies. The only reason for introducing the idea of dark matter was and up to nowadays is the surmised stability of clusters, resulted from their hypothesized negative total energy at the very beginning of their formation. A lot of cunning observations and experiments implemented up to our days did not provide any encouraging results. Nevertheless, the search is going on. It is obvious, that one could decline this idea if refused the mechanism of cosmic objects formation built on the basis of Kant's hypothesis.

\section{Birth of objects according to quantum electrodynamics}

In late 20 s of the last century, researchers knew that photon could appear or disappear during the interaction of elementary particles. Absorption or emission of photons by atoms during the light multiple scattering in atmospheres of stars and planets were under intensive studies and radiation transfer theory was developing rapidly. However, there was no any hint concerning the similar process involving particles possessing of nonzero rest mass. 
Nonetheless, this issue became crucial after the suggestion of the atomic nucleus model by Rutherford (1911). Atomic nuclei in this model consisted of protons and electrons. Rutherford proceeded from the experimental fact that some nuclei showed $\beta$-decay, and protons could emerge from others. Thus, he arrived at a conclusion, that atomic nuclei could contain free protons and electrons. The number of protons, according to Rutherford, provided the mass and atomic number of nucleus and the corresponding number of electrons balanced its electric charge, neutralizing the excess of protons total charge. Rutherford's model seemed to be corresponding to observable characteristics of atomic nuclei. However, detailed studies of atomic nuclei showed later on, that the model led to certain paradoxes. One paradox followed from the uncertainty principle requiring very powerful interaction between protons and electrons to provide the nucleus stability. No experiments ever showed such interaction. Another one called "the nitrogen catastrophe", and related to the spin of the nitrogen nucleus, was also impossible to resolve in the frame of the suggested model. The point is that according to the model of Rutherford the nucleus ${ }^{14} \mathrm{C}$ should show physical behavior of a fermion while empirical data argued in favor of Bose statistics.

Ambartsumian and Ivanenko were the first to solve these paradoxes. They put forward the idea that no free electron could exist in the atomic nuclei and "the whole phenomenon is completely analogous to the emission of photons by an atom, i.e., the electron, like a photon (quantum of light) has no individuality inside the nucleus prior to its ejection" (Ambartsumian \& Ivanenko, 1930b). Moreover, they suggested in the same paper that atomic nuclei might consist of protons and some uncharged unknown elementary particles of nearly proton's mass. That was two years prior to neutron discovery by Chadwick.

That was the second paper of the same authors devoted to the problems of elementary particles. The first one published in the same year (Ambartsumian \& Ivanenko, 1930a) did not pursue a particular issue like the structure of atomic nuclei (if one considers it particular). In the first paper, the authors succeeded in showing that not only mass-less particles can occur and disappear due to interactions but also the particles possessing rest mass have the same properties. The conclusion they arrived at undoubtedly became one of the fundamental results, which highly supported the further development of quantum electrodynamics.

\section{Birth of objects according to quantum electrodynamics}

Ambartsumian (1947) was the first to draw attention of the scientific community to stellar associations, as a distinct class of stellar aggregation. The main structural 
features of stellar associations Ambartsumian listed as followed. Their surface density is smaller, compared with the density of galactic stellar field. On the other hand, the partial concentration of stars belonging to certain spectral classes makes them prominent. In stellar associations always are stars with matter outflow, frequently showing P Cyg type spectral line profile. In some cases, there are open stellar clusters in the center of these systems.

He also made some important conclusions by analyzing the kinematics of the Galaxy. Considering the movement of a stellar association stars in the gravitational field of the Galaxy, one can easily find that the Galaxy differential rotation makes mutual distances of stars larger leading thus to the expansion of these systems in the Galaxy plane. If there are no other mechanisms, which expand the stellar system in the perpendicular direction stellar associations should look like highly flattened. However, the shapes of all the studied stellar associations argue in favor of their isotropic expansion. Therefore, one arrives at a conclusion that besides the differential rotation of the Galaxy there is a kind of inner physical mechanism expanding stellar associations. Moreover, the intrinsic expansion effect should play bigger role than differential rotation effect affects.

The conclusion on the isotropic expansion of stellar associations, in our view, was one of the important results of the observational data analysis. We would like to mention especially the sophisticated estimate of expansion velocity suggested by Ambartsumian. Limitations put to the rate of expansion are coming from the requirement of the isotropy in shape and the fact that this effect of expansion remained unnoticed in terms of radial velocities. The point is that for surpassing the effects of the Galaxy differential rotation, one should expect that the expansion rate is above $1 \mathrm{~km} / \mathrm{sec}$ and, on the other hand, the velocities cannot be above $10 \mathrm{~km} / \mathrm{sec}$, otherwise radial expansion is easily detectable. Therefore, he arrived at a conclusion that the expansion velocity was about $5 \mathrm{~km} / \mathrm{sec}$. This estimate provides about $10-20$ million years for the ages of stellar associations.

Surprisingly the researchers, criticizing conclusion on the expansion of stellar associations, usually forget the arguments relevant to the isotropic shape of these systems. However, those arguments played decisive role in build-up of the new concept on the existence of younger stars. Expansion effects and the velocity estimates were the main tools to lead to the stellar age estimate. Evidently, that was the first outstanding result speaking distinctly in favor of star formation processes in our epoch. The series of papers mentioned above finally initiated intensive research in the new scientific field of studying the newborn stars and star formation regions.

Nowadays it seems clear that Kant-Laplace cosmogonic hypothesis adopted, as the dominant concept for the cosmic objects formation, is the only conceptual 
barrier requiring the rejection of expansion possibility. For the adherents of the mentioned ideology it is more than clear that the mechanism of star formation from a protostellar cloud a negative total energy for the star forming cloud is required. Hence, any bearer of this concept cannot take seriously the idea that a group of newborn stars possesses of positive total energy. One has to consider only selfconsistent physical pictures while discussing the building blocks of that or stay within the same physical frame constructing any physical mechanism.

On the other hand, a concept built on wrong or untenable initial conditions, which fits the empirical or observational data at the beginning, will disagree with new data in the course of their revealing. It resembles the approximation of unknown function by a polynomial, which gives good approach while remaining within the range of given values but diverges violently everywhere out of that range.

In this field of research, an essential clarification of situation one might expect before long, since the astrometric accuracy of orbital observatory Gaia is high enough to reveal at least for nearest associations the expansion velocities estimated by Ambartsumian seven decades ago. If no expansion is registered, undoubtedly one is entitled to strengthen the base for rejection of Ambartsumian's concept. Does it mean that the revealing of expansion rehabilitates the concept rejected for decades? History of science shows that such a continuation is not evident. The inertia of mentality is rather strong, and one always tries to find an explanation for encountered breach of established conceptions by empirical and observational data in the familiar frame of thinking. Nevertheless, in the case of stellar associations the observational accuracy allows already precious implementation of a dedicated program for this purpose. Unfortunately, any similar observations are impossible for extragalactic objects, and for solving of this kind of problems, one should invent some ingenious approaches.

\section{Galaxy formation}

In 1950s, Ambartsumian for the first time suggested a new scenario for the process of galaxy formation (see Ambartsumian 1955, 1956a, 1956b, 1958).This series of analyses he initiated starting studies of multiple systems of galaxies since the multiplicity of objects in any such system might characterize its deviation from the thermodynamic equilibrium. On the basis of simple statistical estimates, he arrived at a conclusion at the very beginning that the relative number of multiple systems within any given cluster of galaxies exceeds many times the one expected for thermodynamically balanced systems(Ambartsumian 1955). He reported his view on the problem at the IAU symposium No 5, held in Dublin. This talk was published also 
by the Armenian academy of sciences in Russian in the same year. That was the first but not the only statement leading the author to the necessity for putting to use a new concept on galaxy evolution completely inconsistent with previous ideas and scenarios. One might consider the paper mentioned above as a brief enumeration of the relevant systems and views on the base of which the author finally established the backbone of the new idea.

We mentioned above the main physical motivation, which served a basis for the series of studies. First, he claimed that the relative number of multiple systems among the all galaxies within any given cluster of galaxies exceeds many times the one expected for the thermodynamic equilibrium. This fact without any additional assumption leads to the conclusion on the joint formation of member galaxies in multiple systems. On the other hand, the analysis of multiplicity type shows that, in contrast to stellar ordinary multiplicity, the high majority of multiple galaxies belong to the Trapezium type systems possessing positive total energy. Hence, at least some of these multiple systems formed recently and are diverging at present. One obtains the same result analyzing differences of radial velocities of binary galaxies.

One of the essential issues he considers in the mentioned first paper devoted to the problems of extragalactic astronomy, in our opinion, is the comparison of needed $M / L$ ratios for binary, galaxies, multiple systems and clusters of galaxies to suppress the kinetic energy of galaxies and keep these systems dynamically balanced. One can find easily now, the higher the multiplicity the larger $M / L$ ratio is necessary for this purpose. In other words, the highest mass to luminosity ratio one needs to provide equilibrium require clusters of galaxies - the systems of galaxies having the highest multiplicity. That is why the idea of dark matter initially appeared when Zwicky $(1933,1938)$ was studying the clusters of galaxies.

However, one should keep in mind always that the requirement of being in the dynamical balance for gravitational objects and their systems actually comes from the dictate of Kant-Laplacian concept of cosmic objects formation only. There are no other serious reasons to expect them being in dynamical equilibrium. The situation is repeating the one we discussed for the stellar associations. The only difference, one might point out, comes from the diversity of geometric scales, bringing its fingerprints also onto mass and time scales describing the objects of the given hierarchical level.

One can find a series of papers by Ambartsumian with considering the physical picture of processes occurring in multiple galaxies. However, one of his papers entitled "On the evolution of galaxies" could serve an introduction for the idea of galactic nuclei activity (Ambartsumian, 1958). It is worth mentioning, that Ambartsumian published his original papers in the little known for the international 
astronomical community journals of Armenian Academy of Sciences, though one could become acquainted with the new ideas thanks to his reports in international meetings. The one mentioned above one could find in the proceedings of Solvay Conference held in 1958.

Actually, that one is kind of survey of his own papers in this field which represents in detail all the observational data used by the author, which led him to the formulation of new approaches and ideas. Unfortunately, the new generation of astronomers actively working in the field of extragalactic astronomy and dealing with the ideas of activity in galactic nuclei, have a few or no notion of argumentations used in late 50 s of last century for basing the idea about the activity of galactic nuclei. Moreover, no any topic survey on the AGN phenomenon prepared during last decades mentions Ambartsumian's contribution to this aria. In their turn, the pioneer ideas, very fruitful for establishing the concept of $A G N$, which are not proved of being useless, are forgotten.

We would like to emphasize again that the main idea concerning the formation of objects from huge bunches of superdense matter met insurmountable obstacle since no gravitational theory could predict the stable existence of such formations. The modern theory of gravitation insists on their inevitable turning into black holes. Therefore, it is obvious that one could not expect any further development of the mentioned concept if one stays in the framework of classical physics.

\section{Nowadays progress in the old problem}

It seems now that one can find new solutions for the Ambartsumian's concept using completely new possibilities opened owing to revealing of the Universe accelerated expansion. Actually, it should completely change the dominant ideas dealing with the cosmic objects formation process. No doubts, it would, if the revealing of the acceleration phenomenon apprehended in a more comprehensive manner and declined some odd stereotypes dictating the main line of thinking. There are some prerequisites listed here for further considering the changes one expects owing to the mentioned discovery.

First, one should consent to the fact that the dark energy, taken as primary source implementing the Universe acceleration, interacts with the ordinary baryonic matter (Statement A). No any exotic scenario invented can help one to refuse this conclusion. If there is no interaction between the dark energy and the baryonic matter no any acceleration of the Universe expansion might be revealed. 
Second, the dark energy fills all the space and is not prerogative of only the large scales (Statement B). No any valid explanation exists to show why it should affect large distances and remain indifferent for the smaller scales. Hence, nobody can indicate the boundary where the effect of dark energy begins to exhibit. Moreover, there is no any logical reason for discussing the issue of space discontinuity of the dark energy. One can suggest that for the homogeneous distribution of dark energy its total amount decreases when one considers smaller volumes. Therefore, it is very hard to reveal its influence at short distances. Nevertheless, one can find its fingerprints if not constrained by dominant ideas forbidding alternative thinking (Harutyunian, 1995; 2011; 2016).

Third, what we call recognizable matter, accessible for empirical investigation, is the baryonic matter having describable atomic structure (Statement C). All other forms of matter (including dark matter) are only mental inventions but unavailable for empirical (including observational) studies. However, calculating any gravitational configurations, researchers pay attention only to the mass of modeled baryonic objects, but not to the atomic structure of matter. On the other hand, atomic structure of matter is its main feature. One of the key features of atoms is the defect of mass, providing their nuclei stability.

Another key feature is that the defect of mass, namely, the part of mass lost by baryons in a nucleus changes from one nucleus to another. In other words, one might establish the fact that elementary particles indistinguishable from the viewpoint of quantum physics, appear in various nuclei with various masses. Actually, this is very important property of baryons, meaning, that their mass in atomic nuclei depends on physical conditions. Then an essential question arises in connection with this: are these conditions changeable for the given nucleus or no any change can happen. Up to nowadays the adopted scientific doctrine asserts that no atomic nucleus we know changes its physical properties. One should consider this issue in detail taking into account the relevant physical laws and regularities based upon available knowledge.

Let us consider now the Statement $A$ in the light of modern physics involving Statements $B$ and $C$ as well. One should recall that according the dominant ideas all the baryonic objects and their systems, including atomic nuclei possess of negative or zero total energy. For atomic nuclei, this lack of energy is the compensating the defect of mass "nuclear binding energy", which does not change for any given nucleus according to quantum theory. However, if there is interaction between the carrier of dark energy and baryonic objects one might consider the issue of energy exchange owing to interaction. One of the general laws of physics is the second law of thermodynamics certifying that the entropy of whole system should increase. 
Taking into consideration that the dark energy is purely positive and the total energy of baryonic objects is negative or zero, one arrives at a conclusion that last ones obtain additional energy due to this interaction.

Although the modern quantum physics rejects any idea concerning the change of nuclear binding energy, the logics and the physical intuition suggest the opposite. One should comply that physical interaction leads to the energetic equilibrium and this process always takes place with continuous redistribution of energy between interacting particles. Let us accept now, that the physical essence of the dark energy is a subject of the quantum world, which is able to change the space with quantum baryonic objects. In this frame of casual determinacy, one arrives inevitably at a conclusion that the binding energy of atomic nuclei decreases in the course of the Universe evolution thanks to the work the dark energy implements at all hierarchical levels. Then one should consider the consequences of such, let us say, thought experiment.

This process leads to the growth of nuclear mass for all nuclei, accompanied with the decrease of their stability. Then one concludes that in the past of the Universe the atomic nuclei we know were comparatively less massive and more stable. Moreover, it seems plausible, that the nuclei showing various types of radioactivity at present were stable in the past, and there existed other radioactive nuclei consisted of much more baryons of much less masses. Continuing the virtual excursus into the past keeping the same logic for the baryonic matter transformation, one arrives at a conclusion that the dipper in the past, the less should be the total mass of baryons, provided that the number of baryons remains constant.

It does mean that the mass of the ordinary (baryonic) matter in the past was much less if comparing the objects consisted of the same number of baryons. It seems more plausible that considering the amount of baryonic matter one should proceed from requirement of the baryon number conservation law rather than from the mass conservation law. Hence, speaking about the hypothetical superdense matter, one should refer to baryons' number density but not to its ordinary mass density. The point is that the mass is changeable and it can transform into energy and vice versa. Therefore, if there can exist some clumps of baryonic matter, delayed in their evolution, one might conclude that getting to normal (for our era) conditions they will rapidly increase their mass to fit the conditions.

This possibility solves the problem of superdense matter. The "old matter" existing in the center of massive objects, presumably composed of huge number of baryons, actually may possess of negligibly small mass. It obtains its real mass corresponding to the nowadays mass-energy balance when ejected into, let to say, present day space, where atomic nuclei are described by Mendeleev periodic table 
and the known abundances. This process should go on in a way to increase baryons mass and decay the heavy nuclei. Moreover, one should expect then this process accompanied with a huge energy release as it takes place when known radioactive nuclei decay, although the amount of energy release in this case will surpass the known decomposition reactions.

By the way, this suggestion helps to solve some paradoxes existing for rather long time. One is associated with the mass creation during the Big Bang hypothesis. All the contemporary theories based on the mentioned hypothesis agreed that all the matter exists since the very beginning after the grand explosion. However, no any explanation exists why the Universe started and continues its expansion. It is clear, that the mass of the Universe was within the Schwarzschild radius, and it did not have any chance for expansion according to laws of modern physics. This conclusion does not depend does one believe in Big Bang hypothesis or not. This is only coherent application of physical laws to analyze very briefly the situation long used hypothesis. Contemporary physics predicts existence of a plenty of black holes at all hierarchical levels of matter autoregulation. Most of astrophysicists believe in the real existence of these hypothetical objects and are sure also that many of these hypothetical objects have primordial origin. Of course, the black holes' subject is a separate theme and we will not consider it here. However, we would like to emphasize one key issue. Since their first prediction by John Michell at the end of the eighteenth century, no any direct evidence appeared yet proving their existence. Therefore, taking also into account the reasoning above, one might suggest a scenario where any matter aggregation goes in a self-consistent way to prevent formation of singularities owing to the structural changes. It seems to be more natural than accepted scenarios of gravitational monsters formation. We will consider this issue in more detail in one of the next papers.

\section{Conclusion}

Ambartsumian suggested his cosmogonic concept on the formation of stars and galaxies about 60-70 years ago. The approach for analyzing the cosmogonic problems was methodologically different from the one adopted by the scientific community. This different approach allowed obtaining qualitatively new results in regard of cosmic objects' evolution process. Moreover, this concept, which he derived completely from the observational data, successfully created very strong philosophical basis as well, since for the first time the author considered the continuity of objects' formation process. 
However, we may establish the fact that the scientific community was unready for apprehending adequately the significance of the suggested concept. There was no sufficient theoretical base for new ideas and, in addition, observational data allowed apply even controversial views for their interpretation of the same phenomena because of the scanty measurements. Nevertheless, in contrary, in the same situation the black hole concept unexpectedly begun flourishing since $90 \mathrm{~s}$ of the last century.

In our opinion, the situation in this field of science bears a strong resemblance to the Ptolemaic model of the world, which provided rather good results on whole, but missed a little here and there. That was a model, which served for about one and a half millennium - incredibly long time for a model constructed on a wrong basis. However, at time of its usage the Ptolemaic model was the only working system giving acceptable results for practical purposes, although it was conceptually incorrect, and the heliocentric model suggested by Aristarchus of Samos existed long before it. Therefore, giving good results on whole is only necessary condition for any model but is not yet sufficient for acknowledging it as a correct one. Speaking straight about present-day cosmogonic models, one cannot guarantee that the adopted concept of cosmic objects' formation, which seemingly "gives good results in whole" leaving some empty rooms in the whole picture, corresponds adequately to the really going on evolutionary processes.

In fact, the only obstacle, which stipulated for rejecting the key idea in Ambartsumian's concept, was impossibility of modelling high mass aggregations of superdense matter in the frame of modern gravitational theory. Now it seems more likely that the theoretical discrepancy occurred not because of the lack of the concept, but it only shows the limitations of the theory and the gravitational models used. Considering consistently the observational data and known features of the baryonic matter one arrives at a conclusion that one should take into attention also the key structural features of baryonic matter while modeling gravitational configurations. The most essential feature to take into account, we are sure, is the atomic structure of the baryonic matter with its concentration in atomic nuclei. The atomic nuclei play a role of energy-to-mass transformation stabilizers in the course of the Universe evolution, using for that the mass defect change mechanism. We argue that interaction of the baryonic matter with the carrier of dark energy inevitably leads to the decrease of mass defect. Therefore, one concludes inevitably that deep in the past of the Universe the mass defect was much larger and consequently the mass of baryons in atomic nuclei - much less. All the processes taking place in the expanding Universe and all the forms of matter, including the energetic forms participate in a self-consistent process of global evolution. 


\section{References}

Ambartsumian, V.A. 1947, Stellar Evolution and Astrophysics, Yerevan (in Russian) Ambartsumian, V.A,1955, Acad. Sci. Arm. SSR, Yerevan (Report at the IAU Symposium 5, ed. N.G.Roman, Cambridge University Press, 1957)

Ambartsumian, V.A. 1956a, Proceedings of Acad. Sci. Arm. SSR, series of Physical Mathematical, Natural and Technical sciences, 9, 23

Ambartsumian, V.A. 1956b, Doklady Acad. Sci. Arm.SSR, 23, 161

Ambartsumian, V.A. 1958, in "La Structure et I'Evolution de 'Univers, Institute International de Physique Solvay", ed.R. Stoops, Bruxelles, 241

Ambartsumian, V.A.; Ivanenko, D.D. 1930a, Doklady Acad. Sci. USSR, A, No.3, 45

Ambartsumian, V.A.: Ivanenko D.D. 1930b, Doklady Acad. Sci. USSR, A, No.6, 153

Harutyunian, H.A. 1995, Astrophysics, 38, 374

Harutyunian, H.A. 2011,Astrophysics, 54, 290

Harutyunian, H.A. 2016, in "Astronomical Surveys and Big Data", ASP Conf. Series, 505, eds. A.Mickaelian, A.Lawrence, T.Magakian, 152

Rutherford, E. 1911,"The Scattering of $\alpha$ and $\beta$ Particles by Matter and the Structure of the Atom", Philosophical Magazine. Series 6, vol. 21. May 1911

Zwicky, W. 1933, Helvetica Physica Acta, 6, 110

Zwicky, W. 1937, ApJ, 86, 217 